\title{
Minitransplants: allogeneic stem cell transplantation with reduced toxicity
}

Running tittle : Minitransplants

\author{
Y. Beguin and F. Baron
}

Department of Medicine, Division of Hematology; University of Liège, Liège, Belgium.

Address for correspondence:

Yves Beguin, MD

University of Liège

Department of Hematology

CHU Sart-Tilman

4000 Liège

Belgium

Tel +32 - 4 - 3667201

Fax +32 - 4 - 3668855

E-mail : yves.beguin@chu.ulg.ac.be 


\section{ABSTRACT}

Allogeneic hematopoietic stem cell transplantation (HSCT) is used for the treatment of selected hematological malignancies. Its curative potential is based on two very different mechanisms, involving the conditioning regimen and the graft-versus-host reactions, respectively. The high-dose chemo-radiotherapy conditioning regimen is aimed at destroying tumor cells, ablating the host immune system (to prevent rejection) and eliminating the host bone marrow (to "make space" for donor stem cells). However, the definitive eradication of tumor cells is also largely mediated by an immune-mediated destruction of malignant cells by donor lymphocytes termed graft-versus-leukemia (GVL) or graft-versus-tumor (GVT) effect. However, because of its toxicity, conventional allogeneic HSCT is restricted to younger $(<55$ years $)$ and fitter patients. These observations led several groups to set up new (less toxic) transplant protocols based on a two step approach : first the use of immunosuppressive (but nonmyeloablative) conditioning regimens providing sufficient immunosuppression to achieve engraftment of allogeneic hematopoietic stem cells and, in a second step, destruction of malignant cells by the GVL effect. These transplants are called nonmyeloablative HSCT or reduced-conditioning HSCT or minitransplants. Preliminary results show that minitransplants are feasible with a relatively low transplant-related mortality (TRM) even in patients up to 70 years. In addition, strong anti-tumor responses are observed in several hematological malignancies as well as in some patients with renal cell carcinoma. As the benefits of minitransplants over alternative forms of treatment remain to be demonstrated, this strategy should be restricted to patients included in clinical trials. 


\section{INTRODUCTION}

The curative potential of allogeneic hematopoietic stem cell transplantation (HSCT) is mediated not only by the eradication of malignant cells by high-dose chemotherapy (and total body irradiation), but also by an immune-mediated graft-versus-leukemia (GVL) or graft-versustumor (GVT) effect $(1,2)$. The power of the GVL effect and its apparent mediation by donor lymphocytes led several groups to infuse donor lymphocytes (DLI) in patients with relapsed leukemia after HSCT $(3,4)$. The induction of durable remissions by DLI demonstrated that the GVL effect is capable of eradicating hematological malignancies even in the absence of chemotherapy. This prompted the introduction of new protocols based on the development of a GVL reaction after low-dose (less toxic) nonmyeloablative preparative regimens providing sufficient immunosuppression to achieve engraftment of allogeneic hematopoietic stem cells (figure 1).

\section{THE GVL EFFECT}

The existence of a GVL effect in humans was first demonstrated by the Seattle's group that evidenced a reduced relapse rate in patients with acute (1) and/or chronic (2) graft-versushost disease (GVHD). This was confirmed by others groups that observed an increased risk of relapse after T cell-depleted (TCD) allogeneic HSCT as well as after syngeneic HSCT (5). The GVL effect was also demonstrated by the evolution of minimal residual disease post-transplant, which often ceases to be detectable only 6 to 12 months after HSCT and by the occurrence of GVL activity with or without GVHD after cessation of GVHD prophylaxis for post-transplant relapse (6).

This led several groups to infuse donor lymphocytes (DLI) in patients with relapsed leukemia after HSCT $(3,4)$. DLI induce a complete remission in about $65 \%$ of the cases in chronic myelogenous leukemia (CML) and in 20 to $30 \%$ of the cases in acute myelogenous leukemia (AML) or myelodysplasic syndromes (MDS) (3,4). Some patients with acute lymphoblastic leukemia (ALL), chronic lymphocytic leukemia (CLL), Hodgkin's disease (HD), 
non-Hodgkin's lymphoma (NHL) as well as multiple myeloma (MM) have also responded to DLI or discontinuation of immunosuppressive therapy $(3,4)$. A GVT effect has also been demonstrated in breast cancer (7) and in renal cell carcinoma (RCC) (8), and possibly in ovarian (9) and non-small cell lung (10) carcinomas.

However, the GVL effect mediated by DLI needs time : the median time to achieve a cytogenetic remission was 85 (range 28 to 241) days for patients with CML (the time to achieve molecular remission can be prolonged) and 34 (range 16 to 99) days for patients with AML (11). Complications of DLI include acute and chronic GVHD and transient marrow aplasia. It is possible to reduce the risk of GVHD without impairing the GVL effect by CD8 depletion of DLI (12) or by starting with a low dose of $\mathrm{T}$ cells and increasing the dose in a stepwise fashion in case of no response (13).

\section{ROLES OF THE CONDITIONING REGIMEN}

Allogeneic HSCT was first considered to deliver supralethal doses of chemotherapy and total body irradiation to tumor cells. The beneficial effect of high doses of chemo-radiotherapy is illustrated by the decreased incidence of disease relapse after autologous HSCT compared to conventional therapy alone in several hematologic malignancies $(14,15)$ (figure 2). However, a large part of the efficacy of allogeneic HSCT is mediated by immune-based GVL or GVT effects (figure 2).

Immature progenitor cells occupy defined niches within the marrow stroma in order to obtain the necessary support for proliferation and differentiation. To allow access for donor cells to these niches, it was commonly believed that host stem cells must be eradicated by the conditioning regimen. However, Storb et al recently demonstrated that the graft itself, most likely through subclinical GVH reactions, is capable to create these marrow spaces in the absence of both chemotherapy and bone marrow irradiation (16). 
It is necessary to abolish host defense prior to transplantation to avoid immune- mediated graft rejection caused by alloreactive cytotoxic host lymphocytes or by HLA-specific antibodies. It was commonly believed that the conditioning regimen was critical for ensuring engraftment. However, donor T lymphocytes (and particularly donor CD8 lymphocytes) are also strongly implicated in the destruction of the host immune system (17). Therefore, TCD of the graft as a method to prevent GVHD may have deleterious effects on engraftment (18). Recently, the Seattle's group demonstrated that optimizing postgrafting immunosuppression can also control the host-versus-graft reaction (19). Thus, contrarily to TCD of the graft that prevents GVHD but increases the risk of graft rejection, optimal postgrafting immunosuppression reduces the incidence of both GVHD and rejection.

\section{NONMYELOABLATIVE CONDITIONING REGIMENS}

Because of its toxicity, conventional allogeneic HSCT is restricted to younger patients ( $<55$ years for allograft procedures with HLA-identical siblings and $<50$ years for unrelated donor transplants) without significant organ impairment. Unfortunately, the majority of malignancies potentially cured by allogeneic HSCT and for which a GVL effect has been demonstrated are more frequent in older patients. Thus, it may be important to develop less toxic approaches to allografting that can also be extended to older patients or patients with pre-existing organ impairment (figure 1).

In 1997, Giralt et al. (20) reported the engraftment of HLA-identical allogeneic HSC after nonmyeloablative chemotherapy based on purine analogs. The rationale for using purine analogs (fludarabine or 2-CDA) was their capacity to inhibit the mixed lymphocyte reaction in vitro and to produce lymphopenia and substantial immunosuppression in vivo. Other pilot trials by the same group confirmed these preliminary results and achieved durable engraftment and remissions in some patients with myeloid as well as lymphoid malignancies, with a relatively low TRM (21,22). The Jerusalem's group developed another nonmyeloablative purine analogbased protocol combining fludarabine, ATG and low-dose oral busulfan (23). This allowed 
achieving engraftment and full donor chimerism in the majority of the patients with a low TRM. However, it should be emphasized that many patients included in this study would be considered eligible for conventional allogeneic HSCT. The feasibility of fludarabine-based nonmyeloablative transplant protocols has also been confirmed more recently by several others groups (24-26).

In an elegant canine allogeneic transplant model, the Seattle's group demonstrated that stable mixed chimerism could be achieved using pre-transplant low-dose TBI combined with post-grafting immunosuppression with a combination of Cyclosporine A (CsA) and mycophenolate mofetyl (MMF) and that post-grafting immunosuppression can serve to control both HVG and GVH reactions (19). Complete chimerism was achieved through DLI. Initial experience in humans showed the feasibility and safety of this approach (27). Moreover, major disease responses were observed in more than $70 \%$ of the patients who had measurable disease pretransplant and achieved sustained engraftment (27).

Finally, the Boston's group demonstrated in a murine model (28) and then in humans that mixed chimerism could be induced in HLA-matched (29) or 2 or 3 loci-mismatched (30) allogeneic HSCT by a nonmyeloablative conditioning regimen combining cyclophosphamide, thymic irradiation and ATG.

\section{MIXED HEMATOPOIETIC CHIMERISM}

Minitransplants usually result initially in mixed hematopoietic chimerism (MC) that can be defined as the presence of 1-95\% hematopoietic cells of donor origin (figure 1). This state is characterized by mutual donor-host tolerance (and thus control of both GVH and host-versusgraft (HVG) reactions) while immune responses against other antigens remain normal. The mechanisms involved include central thymic deletion of both donor- and host-reactive T-cells (because both donor and host dendritic cells are present in the thymus of mixed chimera) and peripheral tolerance due to suppressor T-cells (31). 
For the treatment of hematologic malignancies, mixed donor chimerism is not expected to be always curative. It is now well demonstrated that MC is associated with relapse in patients with diseases such as CML. For those patients with hematologic malignancies, MC can thus be converted to full donor chimerism (FC) by DLI (figure 1).

The assessment of hematopoietic chimerism requires more sensitive techniques than conventional cytogenetic analyses because of the availability of only small numbers of dividing cells. The most current techniques are fluorescent in situ hybridization (FISH) with X- and Yspecific probes in case of sex-mismatched transplant and polymerase chain reaction-based assays of polymorphic mini- or micro-satellite markers in case of sex-matched transplant. Other techniques based on restriction fragment length polymorphism (RFLP) are also used.

The evolution of myeloid and lymphoid chimerism after non-myeloablative HSCT may be discordant. Achievement of full donor T-cell chimerism is associated with disease regression (24). Moreover, the Seattle's group recently showed that the level of T-cell chimerism on day 28 predicted for both graft failure and acute GVHD (27), underlying the importance of lineagespecific chimerism analysis.

\section{ENGRAFTMENT AND TOXICITY AFTER MINITRANSPLANTS}

The engraftment rate is related to the intensity of the conditioning as well as the type of transplant. Generally, more intensive conditioning regimens resulted in higher engraftment rates : graft failure rates ranged from $0 \%$ to $20 \%$ of the cases in the Jerusalem's and in the Seattle's studies, respectively $(23,27)$. Moreover, the immune status of the recipient also appeared to be important for engraftment. For example, a high incidence of graft rejection was observed by the Seattle's group in previously untreated CML patients, inducing them to add fludarabine in their “TBI only" protocol for such patients.

Generally, the conditioning regimens used in the setting of minitransplants are well tolerated, inducing little or no grade 3-4 toxicity, even in patients older than 65 years or with concomitant comorbidities. However, there are important discrepancies among the different 
studies, due to the relative intensity of the regimen used, the age of the patients as well as the type of transplant (sibling versus unrelated, HLA-identical versus mismatch). The 200-day transplant-related mortality (TRM) varied from 4\% in the Seattle study (27) (using low dose TBI alone as conditioning regimen in HLA-identical sibling transplants) to $37 \%$ in the Houston's study (22) (using melphalan and purine analog-containing preparative regimens in related or unrelated graft recipients ineligible for conventional transplants). Age and disease status at transplantation remain important prognostic factors for TRM.

In both animal and human studies, the use of less severe conditioning as well as the initial presence of host hematopoietic cells decrease the severity of acute GVHD. These observations predict that acute GVHD may be limited after minitransplants because of the low intensity of the conditioning and the high incidence of mixed chimerism achieved. Indeed, preliminary data suggest that acute GVHD is relatively mild and generally controllable after minitransplants (27). Moreover, acute GVHD is usually delayed and occurs after patients have recovered from conditioning-related toxicities $(24,27)$. However, there are relatively large discrepancies among the different studies. This variability probably relates to differences in the source of stem cells (bone marrow versus PBSC), type of transplant (related versus unrelated), GVHD prophylaxis, use of ATG as well as age of the patient. In fact, acute GVHD is still the leading cause of nonrelapse mortality. Additional DLI are significantly associated with increased risks of acute GVHD (29). However, the time of infusion as well as the dose of lymphocytes given play a major role.

Because of the short follow-up, the incidence and severity of chronic GVHD are still uncertain. However, preliminary trials reported the occurrence of severe chronic GVHD in some cases (21). Moreover, despite such short follow-up, the risk of chronic GVHD was already 74\% in the Seattle's study (27) and 68\% in the Houston's report (22). 


\section{ANTITUMOR EFFICACY}

Although data are too early to definitively assess antitumor effects, preliminary results clearly demonstrate the occurrence of major disease responses in patients with hematological malignancies as well as some solid tumors.

\section{CLL and lymphoma}

Durable complete responses were observed in several patients with refractory nonHodgkin's lymphoma (NHL), Hodgkin's disease (HD) or CLL $(21,30)$. The Boston's group reported the evolution of 16 patients treated with minitransplant after a conditioning regimen combining cyclophosphamide, ATG and thymic irradiation for primary refractory or relapsing resistant NHL, HD or CLL. Complete responses were observed in 7/16 patients (4/11 patients with NHL, 2/3 patients with HD and 1/2 patients with CLL). Similarly, the Jerusalem's group reported on a group of 23 heavily treated high-risk malignant lymphomas (32). There were 12 patients with resistant disease and 11 with partial response, with 5 having failed a previous autologous transplant. Ten of the 23 patients were alive in CR 15 to 37 months after the transplant and the 3-year probability of disease-free survival was $40 \%$. Kottaridis et al. reported on 14 patients with HD or NHL in partial remission $(n=8)$ or with refractory disease $(n=6)(24)$. The conditioning regimen consisted in fludarabine, melphalan and CAMPATH-1H. Five out of the 14 patients experienced a complete response and stabilization occurred in 7 others patients.

\section{$C M L$}

Complete cytogenetic or molecular remissions were obtained in more than $75 \%$ of $\mathrm{CML}$ patients transplanted in chronic phase $(22,24,25,27,33)$. Moreover, some patients with more advanced phase also achieved molecular remission (27). 


\section{Multiple myeloma}

Durable (> 1 year) partial and complete responses were also observed in some patients with multiple myeloma $(25,27,34)$. Badros et al. (34) studied 16 relapsing multiple myeloma patients (10 in refractory relapse, 4 with partial response and 2 with near CR) receiving a minitransplant after conditioning with melphalan $100 \mathrm{mg} / \mathrm{m}^{2}$. After a median follow-up of 1 year, 5 patients achieved and sustained CR, 3 near CR and 4 partial response (PR). Two patients died of progressive disease and 3 died of GVHD without active disease.

\section{$A M L, A L L$ and $M D S$}

Storb recently reported the results of 17 AML patients treated with related minitransplant after conditioning with 2 Gy TBI \pm fludarabine $\left(90 \mathrm{mg} / \mathrm{m}^{2}\right)(35)$. Eight of 10 patients grafted in CR remained in CR after 5-18 months. Moreover, 2/3 patients with primary refractory disease were in remission at more than 20 months. Prolonged remissions in refractory AML patients were also reported by other groups $(22,33)$.

\section{Solid tumors}

In patients with solid tumors, responses were partial and transient in patients with breast cancer or melanoma, whereas some patients with RCC achieved durable complete responses $(8,24)$. Childs et al. recently reported the evolution of 19 patients treated with minitransplant after conditioning with fludarabine and cyclophosphamide for metastatic RCC (8). Ten of the 19 patients enjoyed major responses, including 3 patients with sustained (> 20 months) complete response. These responses occurred 3-6 months after the transplant and usually after cyclosporine discontinuation. Acute GVHD was associated with disease response but, interestingly, one patient had a complete response in the absence of acute GVHD. 


\section{MINITRANSPLANT AFTER A PREVIOUS CONVENTIONAL TRANSPLANT}

Treatment options for patients who relapse or develop secondary malignancies after autologous or allogeneic HCT are limited. In these patients, results of a second allogeneic HSCT are generally poor, primarily because of a high rate of TRM. Recently, the Jerusalem's group studied the feasibility of a second allogeneic HSCT after a nonmyeloablative conditioning regimen (36). Among the 12 patients included, only one died of procedure-related complications, suggesting that low-intensity regimens significantly reduce TRM associated with second transplants. Moreover, the actuarial disease-free survival at 34 months was 50\%. These findings were confirmed by Kottaridis et al. who reported a 14\% TRM associated with an allogeneic minitransplant for disease relapses occurring after standard autologous or allogeneic HSCT (25). We also reported a low incidence of transplant-related mortality in patients receiving a minitransplant after relapsing after a conventional autologous transplantation (37).

For patients with high tumor burden, the Genoa's group studied the feasibility of conventional autologous HSCT followed by a minitransplant 1 to 3 months later (26) (figure 2). The rationale for high-dose therapy followed by autologous HSCT was debulking and the rationale for minitransplant was to induce immune-mediated anti-tumor effects. The rationale for separating high-dose therapy from allogeneic transplantation was to reduce the TRM and the risk of acute GVHD (see above). Preliminary results evidenced the feasibility of this approach with a low TRM (26).

\section{T CELL DEPLETION OF THE GRAFT}

It is now well demonstrated that a conditioning regimen-related cytokine storm plays a major role in the pathogenesis of GVHD. Moreover, in the minitransplant setting, it is well demonstrated that donor lymphocytes given several weeks after the transplant in mixed chimera induce significantly less GVHD than a similar dose of donor T-cells given together with the transplant, without reducing their anti-tumor efficacy (28). We have recently reported that transplantation of CD34-selected allogeneic PBSC after a myeloablative preparative regimen 
followed by pre-emptive CD8-depleted DLI significantly decreases the incidence of acute and severe chronic GVHD as compared with unmanipulated BMT (38). We also investigated the feasibility and efficacy of minitransplants with CD8-depleted or CD34-selected PBSC followed by pre-emptive CD8-depleted DLI given in incremental doses on days 40 and 80 (depleted group). None of the ten patients included in the depleted group versus $3 / 4$ recipients of unmanipulated PBSC and DLI experienced grade II-IV acute GVHD. Most of the patients included in the depleted group were mixed chimera on day 30 but became full donor chimera after CD8-depleted DLI (37).

Kottaridis et al. (25) recently investigated a novel nonmyeloablative conditioning regimen consisting in CAMPATH-1H, fludarabine $\left(150 \mathrm{mg} / \mathrm{m}^{2}\right)$ and melphalan $\left(140 \mathrm{mg} / \mathrm{m}^{2}\right)$. They observed a high engraftment rate (> 97\%) but most of the patients analyzed were mixed chimera. The incidence of GVHD was exceptionally low (5\% of grade II-IV acute GVHD). The authors explain this observation by the use of in-vivo CAMPATH-1H (achieving in vivo T-cell depletion of the graft because of its prolonged half-life in humans) and by the high incidence of mixed chimerism (known to reduce the incidence and severity of GVHD). However, as mixed chimerism may diminish the GVL effect seen in the allograft setting, longer follow-up is needed to clarify if this approach respects the GVL effect.

\section{CONCLUSION}

In conclusion, minitransplants are feasible and can lead to molecular responses. This transplant strategy offers several advantages over conventional HSCT: [1] TRM is reduced; [2] acute GVHD could be less frequent and less severe than after myeloablative HSCT; [3] minitransplant are possible in patients older than 55 or with concomitant comorbidities. Further clinical trials are needed to define more effective strategies to separate GVL effects from GVHD and to compare the relative efficacy of this approach to conventional treatment (39).

The potential indications for a minitransplant include the same disease indications as for a standard transplant but in patients unfit for a myeloablative conditioning regimen because of age 
(> 50-55 to $70 \mathrm{yrs}$ ) or poor clinical condition (infection, organ failure). It is not unlikely that minitransplants could replace standard transplants even in young fitter patients for diseases such as CML. In addition, minitransplants may be used in diseases where GVT effects are more important than high-dose chemotherapy, such as renal cell carcinoma. In the future, the potential of minitransplants to replace a diseased hematopoietic or immune system may also be exploited to cure non-malignant hematological disorders (such as aplastic anemia, thalassemia, sickle cell disease or SCID) or autoimmune diseases (such as rheumatoid arthritis, multiple sclerosis or sclerodermia). Finally, combination of HSC and solid organ transplants from the same donor may provide definitive tolerance toward the transplanted organ and eliminate the need for prolonged immunosuppression.

As the benefits of minitransplants over alternative forms of treatment remain to be demonstrated, this strategy should be restricted to patients included in clinical trials. 


\section{ACKNOWLEDGMENTS}

Frédéric Baron is Research Assistant and Yves Beguin Research Director of the National Fund for Scientific Research (FNRS, Belgium). This work was supported by grants from "La Fondation Bonjean- Oleffe", "Le Fonds de Recherche Scientifique du CHU Sart-Tilman", "L'Association Sportive contre le Cancer" and the National Fund for Scientific Research (FNRS, Belgium). 


\section{REFERENCES}

1. Weiden PL, N Flournoy, ED Thomas, R Prentice, A Fefer, CD Buckner, and R Storb. (1979) Antileukemic effect of graft-versus-host disease in human recipients of allogeneic marrow grafts. N Engl J Med 300:1068-1073.

2. Weiden PL, KM Sullivan, N Flournoy, R Storb, and ED Thomas. (1981) Antileukemic effect of chronic graft-versus-host disease. Contribution to improved survival after allogeneic marrow transplantation. N Engl J Med 304:1529-1533.

3. Kolb HJ, A Schattenberg, JM Goldman, B Hertenstein, N Jacobsen, W Arcese, P Ljungman, A Ferrant, L Verdonck, and D Niederwieser. (1995) Graft-versus-leukemia effect of donor lymphocyte transfusions in marrow grafted patients. European Group for Blood and Marrow Transplantation Working Party Chronic Leukemia. Blood 86:20412050.

4. Porter DL, RH Collins, C Hardy, N Kernan, WR Drobyski, S Giralt, ED Flowers, J Casper, A Leahey, P Parker, and JH Antin. (2000) Treatment of relapsed leukemia after unrelated donor marrow transplantation with unrelated donor leukocyte infusions. Blood 95:12141221.

5. Horowitz MM, RP Gale, PM Sondel, JM Goldman, J Kersey, HJ Kolb, Rimm, AA, O Ringden, C Rozman, and B Speck. (1990) Graft-versus-leukemia reactions after bone marrow transplantation. Blood 75:555-562.

6. Beguin Y, J Collignon, C Laurent, and G Fillet. (1996) Spontaneous complete remission and recovery of donor haemopoiesis without GVHD after relapse and apparent marrow graft rejection in poor-prognosis myelodysplastic syndrome. Brit J Haematol 94:507-509.

7. Ueno NT, G Rondon, NQ Mirza, DK Geisler, P Anderlini, SA Giralt, BS Andersson, DF Claxton, JL Gajewski, IF Khouri, M Korbling, RC Mehra, D Przepiorka, Z Rahman, BI Samuels, K Van Besien, GN Hortobagyi, and RE Champlin. (1998) Allogeneic peripheralblood progenitor-cell transplantation for poor- risk patients with metastatic breast cancer. $\mathrm{J}$ Clin Oncol 16:986-993.

8. Childs R, A Chernoff, N Contentin, E Bahceci, D Schrump, S Leitman, E Read, J Tisdale, C Dunbar, M Linehan, NS Young, and AJ Barrett. (2000) Regression of metastatic renalcell carcinoma after nonmyeloablative allogeneic peripheral-blood-stem-cell transplantation. New Engl J Med 343:750-758.

9. Bay JO, B Choufi, C Pomel, J Dauplat, X Durando, O Tournilhac, P Travade, R Plagne, and D Blaise. (2000) Potential allogeneic graft-versus-tumor effect in a patient with ovarian cancer. Bone Marrow Transplant 25:681-682. 
10. Moscardo F, J Martinez, GF Sanz, C Jimenez, J Cervera, J Sanchis, F Vera, and MA Sanz. (2000) Graft-versus-tumor effect in non-small lung cancer after allogeneic peripheral blood stem cell transplantation. Brit J Haematol 111:708-710.

11. Porter DL. (2001) The graft-versus-tumor potential of allogeneic cell therapy: an update on donor leukocyte infusions and nonmyeloablative allogeneic stem cell transplantation. $\mathbf{J}$ Hematother Stem Cell Res10:465-480.

12. Alyea EP, RJ Soiffer, C Canning, and J Ritz. (1998) Toxicity and efficacy of defined doses of CD4+ donor lymphocytes for treatment of relapse after allogeneic bone marrow transplant. Blood 91:3671-3680.

13. Mackinnon S, EB Papadopoulos, MH Carabasi, L Reich, NH Collins, F Boulad, H CastroMalaspina, BH Childs, AP Gillio, and NA Kernan. (1995) Adoptive immunotherapy evaluating escalating doses of donor leukocytes for relapse of chronic myeloid leukemia after bone marrow transplantation: separation of graft-versus-leukemia responses from graft-versus-host disease. Blood 86:1261-1268.

14. Zittoun RA, F Mandelli, and R Willemze. (1995) Autologous or allogeneic bone marrow transplantation compared intensive chemotherapy in acute myeloid leukemia. New Engl J Med 332:217-223

15. Andre M, M Henry-Amar, JL Pico, P Brice, D Blaise, M Kuentz, B Coiffier, P Colombat, JY Cahn, M Attal, J Fleury, and N Milpied. (1999) Comparison of high-dose therapy and autologous stem-cell transplantation with conventional therapy for Hodgkin's disease induction failure: a case-control study. Societe Francaise de greffe de moelle. J Clin Oncol 17:222-229.

16. Storb R, C Yu, T Barnett, JL Wagner, HJ Deeg, RA Nash, HP Kiem, PA McSweeney, K Seidel, G Georges, and JM Zaucha. (1999) Stable mixed hematopoietic chimerism in dog leukocyte antigen-identical littermate dogs given lymph node irradiation before and pharmacologic immunosuppression after marrow transplantation. Blood 94:1131-1136.

17. Martin P, S Rowley, C Anasetti, TR Chauncey, T Gooley, E Petersdorf, JO Burik, M Flowers, R Storb, FR Appelbaum, and J Hansen. (1999) A phase I-II clinical trial to evaluate removal of CD4 cells and partial depletion of CD8 cells from donor marrow for HLA-mismatched unrelated recipients. Blood 94:2192-2199.

18. Drobyski WR. (2000) Evolving strategies to address adverse transplant outcomes associated with T cell depletion. J Hematother Stem Cell Res 9:327-337.

19. Storb R, C Yu, JL Wagner, HJ Deeg, RA Nash, HP Kiem, W Leisenring, and HM Shulman. (1997) Stable mixed hematopoietic chimerism in DLA-identical littermate dogs given sublethal total body irradiation before and pharmacological immunosuppression after marrow transplantation. Blood 89:3048-3054. 
20. Giralt S, E Estey, M Albitar, K van Biesen, G Rondori, P Anderlini, S O'Brien, I Khouri, J Gajewski, R Mehra, DF Claxton, B Andersson, M Beran, D Przepiorka, C Koller, S Kornblau, M Korbling, M Keating, H Kantarjian, and R Champlin. (1997) Engraftment of allogeneic hematopoietic progenitor cells with purine analog containing chemotherapy: Harnessing graft-versus-leukemia without myeloablative therapy. Blood 89:4531-4536.

21. Khouri I, M Keating, M Körbling, D Przepiorka, P Anderlini, S O'Brien, S Giralt, C Ippoliti, and R Champlin. (1998) Transplant-lite: induction of graft-versus-malignancy using fludarabine-based nonablative chemotherapy and allogeneic blood progenitor-cell transplantation as treatment for lymphoid malignancies. J Clin Oncol 16:2817-2824.

22. Giralt S, PF Thall, I Khouri, X Wang, I Braunschweig, C Ippoliti, DF Claxton, M Donato, J Bruton, A Cohen, M Davis, B Andersson, P Anderlini, J Gajewski, S Kornblau, M Andreef, D Przepiorka, NT Ueno, J Molldrem, and R Champlin. (2001) Melphalan and purine analog-containing preparative regimens: reduced-intensity conditioning for patients with hematologic malignancies undergoing allogeneic progenitor cell transplantation. Blood 97:631-637.

23. Slavin S, A Nagler, E Naparstek, Y Kapelushnik, M Aker, G Cividalli, G Varadi, M Kirschbaum, A Ackerstein, S Samuel, A Amar, C Brautbar, O Ben-Tal, A Eldor, and R Or. (1998) Nonmyeloablative stem cell transplantation and cell therapy as an alternative to conventional bone marrow transplantation with lethal cytoreduction for the treatment of malignant and nonmalignant hematologic diseases. Blood 91:756-763.

24. Childs R, E Clave, N Contentin, D Jayasekara, N Hensel, S Leitman, EJ Read, C Carter, E Bahceci, NS Young, and AJ Barrett. (1999) Engraftment kinetics after nonmyeloablative allogeneic peripheral blood stem cell transplantation: full donor T-cell chimerism precedes alloimmune response. Blood 94:3234-3241.

25. Kottaridis PD, DW Milligan, R Chopra, R Chakraverty, S Chakrabarti, S Robinson, K Peggs, DC Linch, and S Mackinnon. (2000) In vivo CAMPATH-1H prevents graft-versushost disease following nonmyeloablative stem cell transplantation. Blood 96:2419-2425.

26. Carella AM, E Lerma, A Dejana, T Corsetti, L Celesti, R Bruni, and A Bacigalupo. (1998) Engraftment of HLA-matched sibling hematopoietic stem cells after immunosuppressive conditioning regimen in patients with hematologic neoplasias. Haematologica 83:904-909.

27. McSweeney PA, D Niederwieser, J Shizuru, BM Sandmaier, A Molina, DG Maloney, TR Chauncey, T Gooley, U Hegenbart, RA Nash, J Radich, JL Wagner, S Minor, FR Appelbaum, WI Bensinger, E Bryan, ED Flowers, G Georges, FC Grumet, HP Kiem, B Torok-Storb, C Yu, KG Blume, and R Storb. (2001) Hematopoietic cell transplantation in older patients with hematologic malignancies: replacing high-dose cytotoxic therapy with graft-versus-tumor effects. Blood 97:3390-3400. 
28. Pelot MR, DA Pearson, K Swenson, G Zhao, J Sachs, YG Yang, and M Sykes. (1999) Lymphohematopoietic graft-versus-host reactions can be induced without graft-versus-host disease in murine mixed chimeras established with a cyclophosphamide-based nonmyeloablative conditioning regimen. Biol Blood Marrow Transplant 5:133-143.

29. Spitzer TR, S McAfee, R Sackstein, C Colby, HC Toh, P Multani, SL Saidman, D Weymouth, F Preffer, C Poliquin, A Foley, B Cox, DM Andrews, DH Sachs, and M Sykes. (2000) Intentional induction of mixed chimerism and achievement of antitumor responses after nonmyeloablative conditioning therapy and HLA-matched donor bone marrow transplantation for refractory hematologic malignancies. Biol Blood Marrow Transplant 6:309-320.

30. Sykes M, F Preffer, S McAfee, SL Saidman, D Weymouth, DM Andrews, C Colby, R Sackstein, DH Sachs, and TR Spitzer. (1999) Mixed lymphohaemopoietic chimerism and graft-versus-lymphoma effects after non-myeloablative therapy and HLA-mismatched bone-marrow transplantation. Lancet 353:1755-1759.

31. Sykes M. (2001) Mixed chimerism and transplant tolerance. Immunity 14:417-424.

32. Nagler A, S Slavin, G Varadi, E Naparstek, S Samuel, and R Or. (2000) Allogeneic peripheral blood stem cell transplantation using a fludarabine-based low intensity conditioning regimen for malignant lymphoma. Bone Marrow Transplant 25:1021-1028.

33. Nagler A, M Aker, R Or, E Naparstek, G Varadi, C Brautbar, and S Slavin. (2001) Lowintensity conditioning is sufficient to ensure engraftment in matched unrelated bone marrow transplantation. Exp Hematol 29:362-370.

34. Badros A, B Barlogie, C Morris, R Desikan, SR Martin, N Munshi, M Zangari, A Toor, M Cottler-Fox, A Fassas, E Aniassie, S Schichman, and G Tricot. (2001) High response rate in refractory and poor-risk multiple myeloma after allotransplantation using a nonmyeloablative conditioning regimen and donor lymphocyte infusions. Blood 97:25742579.

35. Storb R. (2001) Nonmyeloablative preparative regimens: how relevant for acute myelogenous leukemia? Leukemia 15:662-663.

36. Nagler A, R Or, E Naparstek, G Varadi, and S Slavin. (2000) Second allogeneic stem cell transplantation using nonmyeloablative conditioning for patients who relapsed or developed secondary malignancies following autologous transplantation. Exp Hematol 28:1096-1104.

37. Baron F, E Baudoux, $P$ Frère, S Tourqui, N Schaaf-Lafontaine, R Greimers, C Herens, G Fillet, and Y Beguin. (2002) Nonmyeloablative stem cell transplantation (NMSCT) with CD8-depleted or CD34-selected PBSC. J Hematother Stem Cell Res 11:301-314. 
38. Baron F, J Siquet, N Schaaf-Lafontaine, E Baudoux, JP Hermanne, G Fillet, and Y Beguin. (2002) Pre-emptive immunotherapy with CD8-depleted donor lymphocytes after CD34selected allogeneic peripheral blood stem cell transplantation. Haematologica 87:78-88.

39. Baron F, and Y Beguin. (2002) Nonmyeloablative allogeneic hematopoietic stem cell transplantation. J Hematother Stem Cell Res 11:243-263. 


\section{LEGEND TO THE FIGURES}

Figure 1 : Schedule of minitransplant. Pre-transplant recipient immunosuppression is carried out with either low-dose chemotherapy or chemo-radiotherapy. Patients receive donor HSC on day 0. Post-grafting immunosuppression is carried out with cyclosporine A with or without MMF or MTX. DLI are given 30-100 days after the transplant in case of mixed chimerism and/or residual disease to obtain full donor chimerism as well as eradication of tumor cells.

$\bigcirc$ Cell of host origin. $\bigcirc$ Cell of donor origin.

Figure 2 : Comparison of various approaches to allogeneic HSCT.

A. Conventional allogeneic transplantation. After a complete remission (CR) is achieved through standard chemotherapy, conditioning with massive doses of chemo-radiotherapy further reduces residual disease that is finally eradicated by the GVL effect.

B. Minitransplant. After low-intensity conditioning that has little impact on the tumor, transplantation of allogeneic stem cells and further DLI are responsible for the eradication of the tumor.

C. Conventional autologous transplantation followed by a mini-allotransplant. After initial CR, high-dose conditioning (with autologous HSCT rescue) further reduces residual disease. Then, a minitransplant is carried out. This is the equivalent of performing a standard allogeneic transplant in 2 steps, i.e. a first step of chemotherapy intensification (autologous transplant) and a second step of adoptive immunotherapy (minitransplant). 
Figure 1
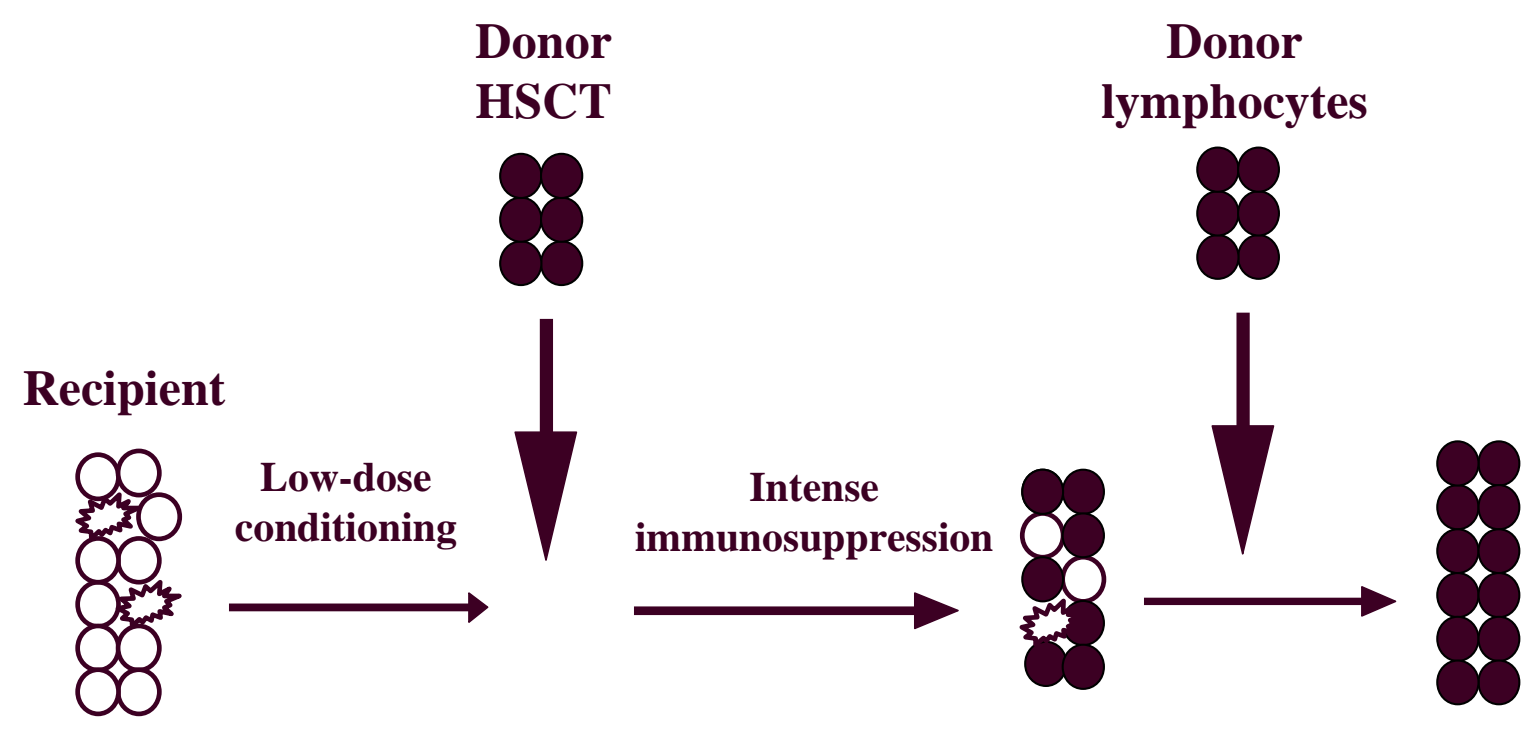

Mixed
chimerism

Full donor chimerism 
Figure 2

A

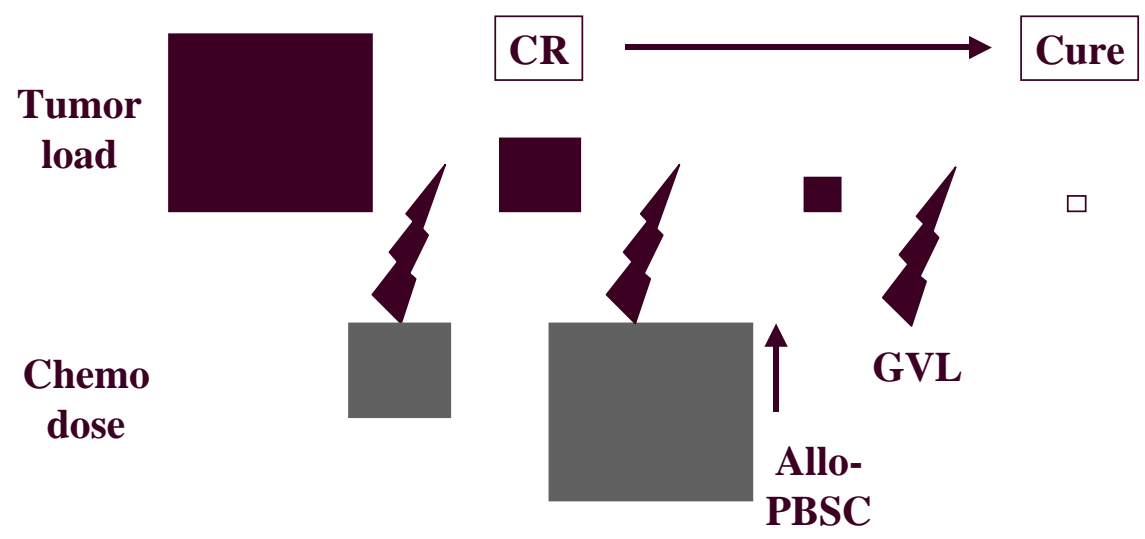

Chemo Allo

B

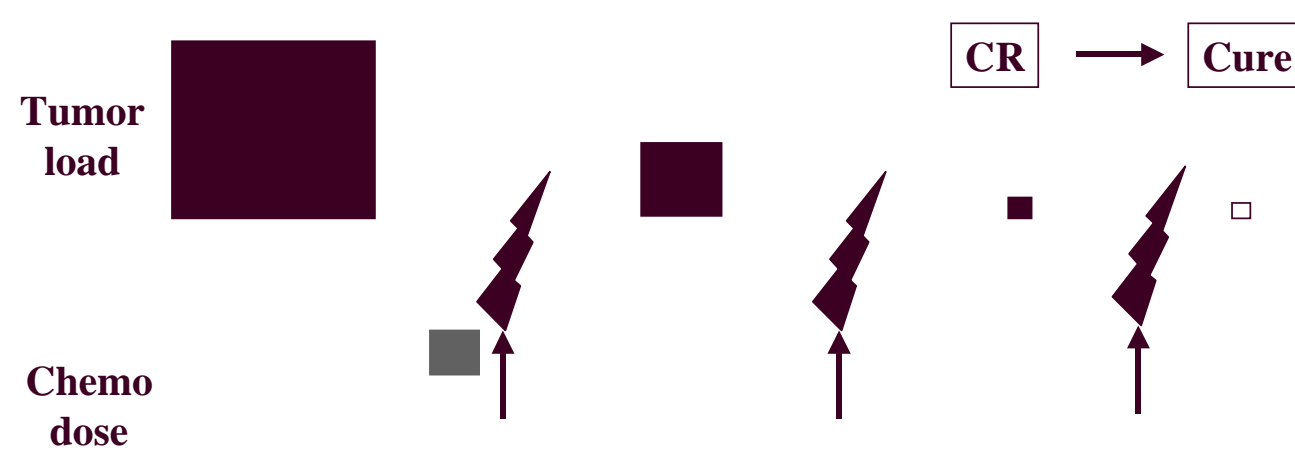

Allo-

DLI

DLI

PBSC

\begin{tabular}{|l|l|}
\hline Mini-allo & DLI \\
\hline
\end{tabular}

C

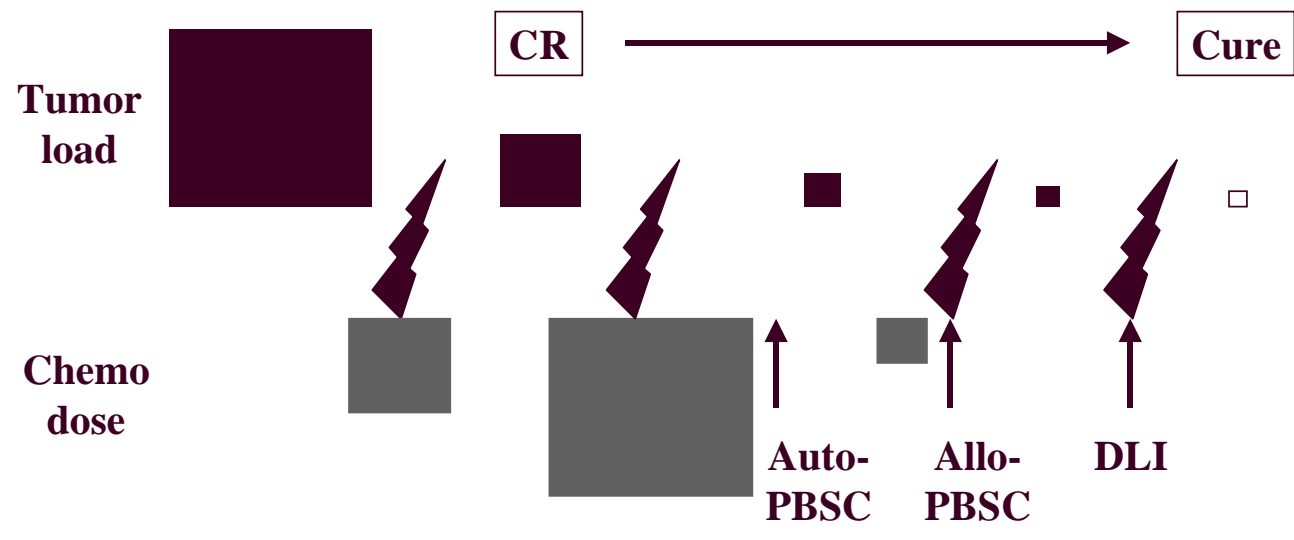

\begin{tabular}{|l|l|}
\hline Chemo & Auto \\
\hline
\end{tabular} 